\title{
Aportes para la revisión y análisis crítico de experiencias de planificación territorial municipal: Colón (Pcia. de Buenos Aires).
}

Contributions for a Revision and Critical Analysis on Experiences on Local Territorial Planning: the case of Colón (Buenos Aires Province).

\section{Gastón W. Cirio}

Becario doctoral del Consejo Nacional de Investigaciones Científicas y

Técnicas (CONICET). Instituto de Investigaciones en Humanidades y Ciencias Sociales (IdIHCS). Centro de Historia Argentina y Americana (CHAyA) y Centro de Investigaciones Geográficas (CIG) Facultad de Humanidades y Ciencias de la Educación (FaHCE). Universidad Nacional de La Plata (UNLP). Doctorando en geografía por FaHCE, UNLP.

gastoncirio@gmail.com

Fecha de recepción:

13.11.15

Fecha de aceptación:

1.2.16

\section{Resumen}

El trabajo constituye una revisión crítica de una experiencia concreta de planificación territorial en la escala municipal, procurando establecer articulaciones entre los abordajes teórico-metodológicos que nutren el debate actual sobre políticas de planificación territorial y la experiencia analizada. El caso de Colón, Buenos Aires, es abordado a partir de tres objetivos específicos: a) describir la experiencia; b) analizar los principios teórico-metodológicos que sustentan las prácticas y elecciones realizadas; y c) ofrecer lineamientos para el debate en torno a los principales nudos problemáticos de la experiencia y los resultados obtenidos. Se combinan para ello, técnicas de análisis documental de múltiples fuentes desde una óptica reflexiva y autocrítica. Por último, se exponen unas breves líneas a modo de reflexiones finales.

Palabras Clave: Planificación territorial - gestión urbana - participación - interdisciplinariedad Municipio de Colón.

\section{Summary}

This article conducts a critical review of a concrete experience of territorial planning at the municipal level, seeking 
to establish its links to the theoretical and methodological approaches that nourish the current debate on territorial planning policies. The case under study is conducted pointing to three specific objectives: a) a description of the specific experience; $b$ ) a discussion on the theoretical and methodological principles underlying the effective policy implementation and choices; c) to provide guidelines for the debate on the main problematic nodes faced during the implementation and in the achievement of outcomes. In order to do this documentary techniques of analysis combine multiple sources from a reflective and self-critical perspective. The article ends with a few final remarks.

Key words: Territorial planning - urban management participation - interdisciplinarity - Municipality of Colón.

\section{Introducción:}

El presente trabajo constituye una revisión analítica y crítica de una experiencia de planificación territorial municipal, procurando establecer articulaciones entre los abordajes teóricometodológicos que nutren el debate actual sobre políticas de planificación del territorio y la experiencia analizada. Así, el objetivo general que se persigue es realizar una revisión crítica sobre la experiencia de formulación del Anteproyecto de Ordenamiento y Uso del Suelo del Municipio de Colón, Provincia de Buenos Aires. Asimismo, los objetivos específicos son tres: a) Describir brevemente la experiencia analizada; b) Analizar, en clave crítica los principios teórico metodológicos que sustentan las prácticas y elecciones realizadas en esta experiencia; c) Ofrecer lineamientos para el debate en torno a los principales nudos problemáticos de la experiencia y los resultados obtenidos. El interrogante que da lugar a estos objetivos y que articula este trabajo puede ser pensado de la siguiente manera: ¿Cuáles son los supuestos teórico - metodológicos, parcialmente implícitos, que están presentes en esta experiencia de planificación, y qué consecuencias técnico-metodológicas, políticas y sociales generan? Se intentará dar una respuesta a este interrogante organizando el texto en tres apartados. En primer lugar se realiza una presentación describiendo el contexto general del trabajo, sus objetivos y el marco normativo vigente en el cual se inserta la experiencia analizada. A continuación se ofrece una revisión crítica de los elementos más significativos del proyecto en torno a cinco puntos: a) La perspectiva de planificación territorial adoptada; b) La conformación del equipo y los recursos; c) La participación social y sus implicancias; d) Los resultados y sus formas de producción; e) La (des)articulación con las instancias de validación, implementación y gestión. Por último se exponen unas breves líneas a modo de cierre. 


\section{Presentación del caso y antecedentes.}

\subsection{El municipio de Colón, Provincia de Buenos Aires.}

El caso que en estas líneas se analiza refiere a un proyecto de investigación territorial cuyo objeto fue la elaboración de un Anteproyecto de ordenamiento urbano y territorial para el Partido de Colón, Provincia de Buenos Aires, Argentina. Se trata de uno de los 135 municipios de la provincia, ubicado en el sector noroeste de la región pampeana bonaerense, con una población actual estimada de 25.000 habitantes (23.206 habitantes de acuerdo al Censo Nacional de Población de 2010). La cabecera de partido, Colón, es una ciudad típica de la denominada "pampa gringa", con un plano en damero español tradicional de 16 cuadras de lado, una gran plaza central y una periferia cuyo proceso de periurbanización en las dos últimas décadas le confiere rasgos distintivos. Se puede distinguir en la ciudad un damero subdividido en cuatro sectores o cuadrantes iguales de ocho cuadras cada uno, con un trazado regular. Del total poblacional cerca del $80 \%$ corresponde a la planta urbana de la ciudad cabecera, mientras que las delegaciones rurales El Arbolito, Pearson y Sarasa se encuentran por debajo de los mil habitantes, con un aproximado de 900, 800, y 700 habitantes respectivamente.

\subsection{Normativa vigente, contexto institucional y objetivos del trabajo.}

Al momento de realización del proyecto que se analiza en este trabajo, el cuerpo normativo que regula el uso y ocupación del suelo del Municipio de Colón está conformado por las Ordenanzas Municipales 252/79 y 1069/89. Dichas ordenanzas comprenden la primera etapa del proceso de planeamiento, en el marco del Decreto Ley 8912/77 de Ordenamiento Territorial y Uso del Suelo de la Provincia de Buenos Aires, que en su Capítulo III art. 75 establece: "El proceso de planeamiento se instrumentará mediante la elaboración de etapas sucesivas que se considerarán como partes integrantes del plan de ordenamiento. A estos efectos se establecen las siguientes etapas: 1.- Delimitación preliminar de áreas; 2.- Zonificación según usos; 3.- Planes de ordenamiento municipal; 4.- Planes particularizados.” Así, las ordenanzas municipales mencionadas establecen una delimitación preliminar de áreas, resultando en un instrumento precario y limitado para la gestión municipal territorial a dos y tres décadas de sancionadas las mismas. En este contexto institucional y normativo, la experiencia, se aboca preferentemente al nivel de la segunda etapa denominada "Zonificación según usos". Sin embargo, se incluyen también algunos aportes relacionados con la tercera etapa: "Planes de ordenamiento municipal”. (Municipalidad de Colón, 2008). ${ }^{1}$

En el marco brevemente presentado, los objetivos principales que el estudio propone son:

"Realizar un diagnóstico territorial que reconozca patrones de ocupación y apropiación territorial, tendencias 
$y$ conflictos identificados en el espacio municipal, lineamientos estratégicos para el corto y mediano plazo en la zona de referencia, de manera de superar los desajustes normativos de la Ordenanza Municipal Nro. 252/79 y Ordenanza Nro. 1069/89, teniendo en consideración la normativa provincial vigente, Decreto Ley 8912/77 - Ley de Ordenamiento territorial y Uso del Suelo y demás leyes provinciales de aplicación. Redactar un Anteproyecto de Ordenanza Municipal de Ordenamiento Territorial y Uso del Suelo en el Partido de Colón, Buenos Aires, en el que se contemple para la elaboración del mismo, conocer el territorio y sus lugares o patrones de ocupación y apropiación territorial; el Partido de Colón, su localidad cabecera, su periferia, su ejido de chacras, sus localidades no cabeceras, su espacio rural.” (Ibid., p. 4)

\subsection{Breve resumen del proyecto y sus características.}

Tal como puede apreciarse en la figura 1, el proceso de esta experiencia puede pensarse como constituido por tres etapas. Una etapa previa al proyecto, en la cual la desactualización de la normativa vigente en el lapso de más de dos décadas da lugar al surgimiento de necesidades sociales y de gestión municipal que deben ser abordadas con un instrumental de planificación y gestión acorde a las dinámicas territoriales que se desarrollan en el contexto local y regional en esos años. Esto motiva el llamado público del municipio para la elaboración del anteproyecto de ordenamiento urbano y territorial. La segunda etapa es la del momento del desarrollo, propiamente dicho, de la experiencia que aquí se analiza; transcurre entre 2007 y septiembre 2008. Por último, un momento posproyecto, que transcurre entre 2008 y la actualidad, en el cual se pasa a las instancias correspondientes de validación institucional a nivel municipal y provincial.

El momento de desarrollo del proyecto, a su vez, puede ser pensado como constituido por cinco fases sucesivas e interrelacionadas en las cuales se parte de la presentación pública y el armado del diseño institucional - participativo para llevar a cabo el estudio, hasta la presentación en asamblea abierta del informe final y la normativa instrumentada en un código de ordenamiento.

En el marco de este proceso, el primer paso fue la presentación formal y el armado de las comisiones de trabajo, a través de una convocatoria abierta a la sociedad. El resultado fue la constitución de cuatro comisiones: técnica, política, ciudadana y empresaria, siendo los principales actores del proceso de participación el intendente municipal, concejales, arquitectos y representantes de distintos sectores de la sociedad local. 
El segundo paso fue la realización de entrevistas con funcionarios municipales a cargo de la Secretaría de Planeamiento y Obras Públicas del Municipio de Colón. Los ejes fundamentales trabajados en estas entrevistas fuero: 1-Pre-diagnóstico e imaginario actual, 2-Conflictos y limitaciones, 3- Tendencias, perfiles y proyecciones, 4-Obras y necesidades prioritarias. En complemento, se diseñó un esquema de diagnóstico operativamente diferenciado en un "diagnóstico social" y otro "diagnóstico territorial", en el cual se implementaron distintos métodos y técnicas. Entre las más destacadas en el primero es posible mencionar las entrevistas en profundidad, focus group, talleres participativos, método Delphi y matrices Foda. En el caso del diagnóstico territorial se aplicaron principalmente técnicas de teledetección, interpretación de fotografias aéreas, relevamiento de usos reales del suelo, producción, análisis y correlación de cartografía temática, así como entrevistas semiestructuradas a informantes clave.

Una vez realizados los diagnósticos, con participación activa de distintos actores sociales locales, el proceso dio lugar a la elaboración de unos lineamientos estratégicos preliminares y una propuesta de código. A su vez, estos productos de la investigación realizada fueron puestos a consideración, revisión y validación a través de las distintas comisiones de trabajo. Por último, la reformulación de las propuestas a la luz de las interacciones realizadas en las comisiones, dio lugar a la redacción del informe final, la instrumentación del código y la presentación pública del anteproyecto de ordenamiento urbano y territorial de Colón.

El proceso de investigación, materializado en el informe final, detecta como principal problema la "Ausencia de un modelo de desarrollo de la ciudad; lo que provoca un crecimiento extendido y fragmentado, con conflictos derivados de la incorrecta localización de las actividades" mientras que de dicho diagnóstico se desprenden, articulados, problemas específicos tales como segregación socio - espacial en el sector noreste de la ciudad; conflictos asociados a la circulación masiva de transporte de cargas en la planta urbana; inapropiada y dispersa ubicación de las zonas industriales; falta de vinculación con jurisdicciones vecinales; deficiencias en la provisión de agua potable por problemas infraestructurales en la fuente y falta de capacidad en corto plazo; falta de infraestructuras de servicios básicos como así también de las nuevas infraestructuras de información y comunicación en el área central; expansión urbana residencial no planificada; falta de lugares atractivos para la radicación de establecimientos industriales, perfil de centro sin acuerdos; territorio rural sin suficiente jerarquización vial rural ni de sus tres centros de servicios rurales; baja densidad poblacional; falta de equipamientos sociales adecuados y mala distribución de los existentes; sub-equipamiento del campo y pérdida de relación entre la vida del campo y la ciudad; falta de estrategia de integración de la industria que está asociada a la actividad rural. (Municipalidad de Colón, 2012: 112).

Asimismo, del análisis documental de la experiencia se desprenden los principales conceptos ordenadores de la propuesta de ordenamiento, entre los que destacan Desarrollo 
sustentable, Proyecto social, Lugar, Calidad Urbana y Ordenamiento territorial policéntrico, entre otros. Considerando estas directrices conceptuales, los problemas expuestos y los desafios establecidos, las estrategias generales para el ordenamiento territorial fueron divididas en cuatro apartados: estrategias de movilidad, estrategias socio-urbanas, estrategias socioeconómicas y estrategias ecológicas. En el marco de estas grandes líneas estratégicas, las pautas proyectuales que orientan la propuesta establecen, entre otros, los principios de rejerarquización del sistema vial para la estructuración del territorio y la etapabilización de la propuesta. Sobre este último principio, la propuesta establece cuatro etapas claramente diferenciadas. La primera de estas etapas supone la "sanción de las normas urbanísticas que sirven de soporte jurídico para la instalación de las actividades, la asignación de indicadores urbanísticos, las pautas al volumen de las edificaciones o las normas a la producción arquitectónica y para la generación de los instrumentos de gestión necesarios para la gobernanza" (Ibid., p.119) y es en ella donde se concentra la mayor parte de los resultados de la propuesta analizada. Así, establece dos tipos de instrumentos de planificación principales: a) Zonificación, con todos sus indicadores urbanísticos, y b) Instrumentos de Gestión: estableciendo, entre otros, la Oficina de Planeamiento Urbano y Desarrollo Territorial como autoridad de aplicación, un Consejo de Participación Territorial (CPT), una Comisión Interpretativa del Código (CIC) y un Fondo de Desarrollo Urbano. Por otro lado, las tres etapas restantes son contempladas en la propuesta mediante lineamientos estratégicos de menor grado de operativización, considerando el mediano y largo plazo con una escala local y regional.

\section{Análisis crítico}

A continuación se ofrece una revisión crítica de la experiencia de planificación territorial analizada a partir de una doble vertiente metodológica. Por un lado, a partir del análisis documental de contenido sobre el informe final, así como de informes parciales, material gráfico, cartográfico y sonoro resultante del proceso de investigación. Por otro lado, a partir de una óptica reflexiva y autocrítica se procura complementar el análisis incorporando la perspectiva actual del autor de este trabajo sobre su propia práctica como integrante de un equipo interdisciplinario en el marco del proyecto de investigación e intervención analizado.

\section{2.a) La perspectiva de planificación territorial adoptada.}

En primer lugar parece necesario plantear sintéticamente la complejidad inherente a la conceptualización y abordaje que asume la planificación territorial. Este concepto adquiere diversos alcances y acepciones de acuerdo a los enfoques teóricos, y especialmente, en relación a los periodos históricos a los que refiera. Estas múltiples concepciones de la planificación territorial oscilan entre extremos que pueden considerarla como una herramienta al servicio del capital, responsable de la exacerbación de las disparida- 
des sociales en los países subdesarrollados (Santos, 1979:13), y como una aproximación integrada a la valoración del territorio, desde una óptica de optimización de sus aptitudes al desarrollo de la actividad humana con minimización de impactos ambientales y sociales. (Farinós Dasí, 2000:71).

En ellas se puede observar las dos grandes corrientes de pensamiento en planificación territorial latinoamericana que Coraggio (1994: 203-221) reconoce y desarrolla como principales. Por un lado, propuestas de planificación que pueden enmarcarse en una corriente neoclasicista, de base economicista y fisicalista, en la que se asume que la sociedad debe ser un todo armónico donde las problemáticas "patológicas" deben ser eliminadas mediante la reinstauración de mecanismos de mercado en la búsqueda de un óptimo social. Se presenta en esta corriente una tendencia a la cosificación territorial, donde "la configuración territorial que adopta el sistema demográfico-económico es vista como un problema en sí mismo" (Coraggio, 1994:195). Es posible identificar en este enfoque algunos aspectos clave de la tradicional Planificación Normativa Racional aplicada al territorio. Asimismo, el enfoque de la Planificación Estratégica territorial (Elizalde Hevia, 2003; Vázquez-Barquero, 1995, 2009) aun incorporando nociones sistémicas y de participación ciudadana, no deja de formar parte de esta gran corriente, ya que se considera una "herramienta para racionalizar la toma de decisiones y la gestión de las ciudades y regiones" (Vázquez-Barquero, 2009:5) con un fuerte énfasis economicista y competitivo.

La otra gran corriente de pensamiento es la corriente crítica, nutrida por el pensamiento marxista, que redefine la problemática territorial al considerar que las contradicciones y los conflictos sociales no son situaciones patológicas a modificar, sino parte constitutiva del mismo sistema. En esta corriente se pueden identificar, reconociendo los aportes de Coraggio, cuatro dimensiones constitutivas fundamentales: a) la inscripción de la planificación territorial en un marco de pugna teórico-ideológica en la cual es necesario definir los alcances de la misma, sus características y formas de realización; a su vez, estos debates deben inscribirse en relación a un contexto de proyectos político-sociales también en pugna; b) La generación de conocimiento científicamente fundado sobre la problemática territorial específica de cada sociedad, evitando los “diagnósticos” basados en estadísticas, indicadores e inventarios que nada dicen sobre las relaciones constitutivas del territorio investigado; c) La búsqueda de una formación técnico-profesional acorde al enfoque, lo que supone la generación de visiones integrales alternativas a la híperespecialización positivista, a las investigaciones concentradas en aspectos parciales y a los recortes fragmentarios de la realidad territorial investigada; d) La incorporación de los agentes sociales y sus relaciones de poder a la investigación. Entendiendo que la planificación territorial no es una actividad de intervención externa, sino que se inserta en el interior de los procesos sociales, es necesario incorporar la acción de los agentes sociales explícitamente a la planificación territorial y las relaciones de poder estudiadas como una necesidad imperativa para pensar una planificación territorial desde este enfoque crítico. 
Planteadas estas dos grandes corrientes, se hace evidente que no hay definición apriorística de la planificación territorial y por lo tanto se puede plantear, coincidiendo con la propuesta de Coraggio, la diversidad de planificaciones posibles (Coraggio, 1994: 189). Así, el enfoque que se utiliza en este trabajo se puede considerar como parte de la línea de investigación de evaluación de políticas públicas (Villarreal Cantú: 2010; Aguilar 1992, 2009), pero a partir de un enfoque o marco analítico geográfico-territorial.

Ante esta multiplicidad de perspectivas posibles para la planificación territorial es interesante destacar que en el abordaje que se explicita en el informe final del anteproyecto analizado, aparecen parcialmente elementos propios del urbanismo y el planeamiento físico. Así, aparecen referencias a la "tercera revolución urbana" y a los principios del Nuevo Urbanismo (Ascher, 2007), los cuales procuran ser adaptados a la realidad del caso de Colón, considerando que las diferencias respecto a las concepciones del ordenamiento urbano y territorial son "más pragmáticas que teóricas” (Municipalidad de Colón, 2008:4). En este marco, el abordaje que se asume en el informe incorpora la noción de contexto incierto y la primacía de la planificación - gestión estratégica por sobre la planificación urbana normativa. Por otro lado, la explicitación de la concepción asumida abre algunos interrogantes sobre sus alcances y características:

Respecto a la concepción del ordenamiento y la gestión urbana para Colón, la misma puede resumirse en tres instancias, propias de la "construcción de la ciudad": urbanización, edificación y concentración, cada una de ellas con diferentes formas de regulación. Ello surge del planteo siguiente: en primer lugar, el espacio se urbaniza, luego se construye y finalmente puede concentrar fijos y flujos. Respecto a la concepción del ordenamiento y la gestión rural para Colón se aplica una concepción de ordenamiento territorial y gestión del territorio rural orientada a promover un desarrollo socio-territorial sustentable apoyado en huellas e improntas así como en instancias de creación, expansión y consolidación de los patrones de ocupación y apropiación territorial más significativos del distrito

(Municipalidad de Colón, 2008:7)

Se aprecia un fuerte sesgo fisicalista en estas concepciones, con mínimas referencias a los procesos sociales de baja concreción físico-espacial.Asimismo, la concepción de planificación que atraviesa todo el proyecto, y se encuentra a lo largo del documento final analizado, se caracteriza por un fuerte economicismo vinculado a la competitividad territorial, la integración a redes urbanas especializadas y la generación de instrumentos de atracción del capital. 
Por otro lado, las características del contexto normativo e institucional presentado en el apartado 1.2, las tradiciones técnicas institucionales locales, los requerimientos y las necesidades del municipio demandante del estudio, confieren características restrictivas respecto a las concepciones posibles de asumir en un proyecto como el que aquí se analiza. En ese marco, la concepción de planificación territorial asumida parece ser un híbrido pragmático en el cual se tensionan abordajes estratégicos-situacionales de corte liberal, con elementos y herramientas propias de la planificación normativa racional. Esto no resulta paradójico si consideramos, siguiendo a López (1999) que el urbanismo tradicional y la planificación normativa racional ya contenían aspectos estratégicos en la medida que la planificación vertical siempre dependió de una estrategia productiva determinada y de estrategias de control social necesarias.

A pesar de la incorporación de elementos participativos y mecanismos de gestión no se logra superar ciertas limitaciones legales respecto a las características prescriptivas de las herramientas resultantes -así como a sus pretensiones regulatorias-, y a una visión general que puede ser inscrita en la corriente neoclasicista propuesta por Coraggio. En este sentido, es interesante destacar que la concepción presente en este proyecto parte de una visión integral del territorio de base marxista que descansa en los aportes de Milton Santos (1996). Esta característica aporta elementos parcialmente contradictorios respecto a la planificación territorial en si y su concepción teórica, a la vez que permite introducir una mirada más rica y una metodología más completa para el abordaje de la investigación territorial realizada. Así, es posible identificar en este proyecto al menos en el plano teórico metodológico sobre el territorio, un intento de superar las limitaciones propias de muchas de las experiencias de planificación en Argentina. En apretado resumen, siguiendo los aportes de Szajnberg et al (2010), Garay (2004), Reese (1999, 2006), Catenazzi y Reese (2001), Clichevsky y Rofman (1989), Coraggio (1994) entre otros; estas limitaciones serían: a) incapacidad para desarrollar una comprensión cabal e integral de los procesos territoriales, b) importantes carencias teórico - metodológicas sobre el territorio, c) concepciones fisicalistas y espacialistas, d) visiones intervencionistas y neopositivistas, e) espacio como reflejo de la sociedad,f) intervención y desarrollo mediante vinculación directa y causal., f) abordajes sectoriales y temáticos poco integrales, g) excesiva y artificial especialización de los instrumentos por temáticas (vivienda social, industria, etc.), h) fragmentación de la unicidad del territorio y pérdida de relaciones constitutivas.

No obstante estas buenas intenciones de superación, es necesario señalar que las dificultades para sortear estas limitaciones parecen ser fuertes - y no siempre muy conscientes - lo que redunda en experiencias como la que aquí se analiza. Desde el plano discursivo aparecen elementos teóricos contradictorios que entran en tensión y no logran resolver una metodología de planificación territorial completamente coherente. En este marco, tal como fue mencionado, el resultante híbrido pragmático no parece constituir un planteo sólido que represente completamente alguna línea teórica de planificación 
territorial, sino que contempla elementos diversos en torno a un proyecto, que puede caracterizarse como de planificación física con énfasis urbanístico o municipal (Cabeza, 2012).

\section{2.b) La conformación del equipo y los recursos.}

En el desarrollo de la experiencia de Colón aparecen dos tipos de elementos, en alguna medida relacionados, que han tenido influencia directa en ciertas condiciones resultantes en el proyecto. Por un lado, la disponibilidad, elección y necesidad de determinados recursos. Por otro lado, las condiciones del trabajo en equipo vinculado, como se verá, a las características teórico- metodológicas (2a) y a los sesgos disciplinares.

Respecto a los recursos, parece pertinente diferenciar aquellos referidos a la dimensión temporal, aquellos relacionados a la disponibilidad de recursos económicos - técnicos, y aquellos conformados por los recursos humanos. Sobre la dimensión temporal destaca el acotado periodo de tiempo del que se dispuso entre el inicio formal del proyecto (mediante acuerdo contractual por licitación pública) y la presentación de los resultados del mismo. Considerando que el periodo total de lo que ha sido considerado en este trabajo como "el momento del proyecto"(octubre de 2007 a septiembre de 2008), surge claramente que se trata de un recurso temporal significativamente escaso para un proyecto de planificación de un municipio. Esta situación es aún más compleja y limitante si se considera que el trabajo en campo, en relación a esta disponibilidad temporal total, se vio significativamente acotado, resultando en un intenso trabajo desarrollado en un período menor a los nueve meses. En relación a los recursos humanos, el equipo completo de trabajo estuvo conformado por un total de once profesionales de los cuales nueve de ellos pertenecían al equipo consultor y dos de ellos formaban parte del equipo técnico local a cargo de la Dirección de Planeamiento y Catastro Municipalidad de Colón. A su vez, el trabajo de campo y la mayor parte del proceso estuvo a cargo de cinco de los nueve integrantes del equipo consultor. En este sentido, aparecen ciertas limitaciones con respecto a la disponibilidad de recursos humanos en campo, a la vez que supone reiteradas estadías de trabajo de gran intensidad. Por último, los recursos económicos disponibles, a partir del acuerdo mediante la licitación ya mencionada, resultaron significativamente escasos en relación a las características y ambiciones del proyecto, habiendo repercutido claramente en la disponibilidad de recursos humanos, en la extensión temporal y en los recursos técnicos desplegados en campo para el trabajo.

Respecto al trabajo en equipo se parte de una concepción, al menos discursiva, de trabajo interdisciplinario a partir de la explicitación de este principio a lo largo del informe final: "Para llevar a cabo este diagnóstico se parte del trabajo interdisciplinario llevado a cabo in situ ...” (Municipalidad de Colón, 2008:9). Sin embargo, la articulación interdiscipinaria es, como se sabe, mucho más compleja en la práctica que en el discurso. Así, aparece en esta experiencia la intención de generar un diálogo constante entre los aportes discipli- 
nares de los distintos profesionales participantes en la construcción de una perspectiva interdisciplinar orientada a la planificación territorial.Tal como fue señalado en un trabajo precedente (Cirio, 2012) en las últimas décadas se aprecia un gran esfuerzo por parte de diversos científicos y filósofos por trascender la lógica de formación y abordaje disciplinar (10). La experiencia aquí analizada se enmarca en ese esfuerzo e intención. Sin embargo aparecen elementos de la praxis investigadora y de su materialización en el producto resultante que hacen pensar que las ambiciones interdisciplinares de esta experiencia no han logrado cristalizarse en toda su dimensión. En este sentido, las limitaciones y problemáticas vinculadas al diálogo entre profesionales de la arquitectura, la ingeniería, la geografía, y la comunicación social en torno a una misma problemática han resultado en una suma de perspectivas que lejos de trascender la visión parcial y sesgada sobre problemáticas comunes, ha constituido en muchos puntos conflictos conceptuales de dificil superación. No obstante la buena intención, se constata en este trabajo una suma de visiones, métodos y técnicas más propias de la visión disciplinar que en todo caso puede caracterizarse como multidisciplinar. Asimismo, respecto a la escasa diversidad disciplinar al interior del equipo, se entiende que esta es reflejo del abordaje de planificación física con énfasis urbano municipal ya referido, no resultando azarosa la ausencia de economistas, sociólogos, antropólogos, entre otros posibles profesionales pertinentes.

\section{2.c) La participación social y sus implicancias.}

Se ha señalado a lo largo del trabajo que la incorporación de mecanismos democráticos de participación en la experiencia de planificación que aquí se analiza son propios de una perspectiva estratégico-participativa, que constituye parte del acervo puesto en práctica en el caso de Colón. Sin embargo al analizar las modalidades, los alcances y las características cuantitativas y cualitativas de dicho proceso participativo se constata que la experiencia de Colón comparte muchas de las limitaciones señaladas por los autores referentes en la materia. En este sentido López (1999) analiza en profundidad la dimensión social y política en relación a la participación de colectivos dominados en los procesos de planificación. Al respecto, señala que ni la planificación estratégica ni la planificación normativa resuelven estas asimetrías ni resuelven los conflictos sociales asociadas a ellas. Coincidiendo y continuando con los postulados de este autor, puede verse en el caso de Colón que se asume un

paradigma posmoderno neoliberal de participación como consenso y compromiso (o contrato) sin resolver los conflictos ni las numerosas exclusiones de colectivos que no son convocados a los procesos de planificación

(López, 1999: 113) 
Así, el proceso participativo analizado se caracteriza por la presencia mayoritaria de sectores sociales con organización previa al proyecto, con fuertes componentes corporativos (colegio de arquitectos, martilleros, empresarios locales, funcionarios públicos, etc.) y una significativa minoría de parte de ciudadanos no organizados, organizaciones de base territorial y sectores populares en general. En el mismo sentido crítico acerca de la participación ciudadana en este tipo de instrumentos de planificación los trabajos de Garay (2004), Reese (1999, 2006) y Chiarella Quinhoes (2005) entre otros, dan cuenta del carácter centralizado y excluyente respecto de los intereses y capacidades de los distintos actores territoriales que participan de estas experiencias, tratándose, en muchos casos de procesamientos políticos "escenográficos". En este sentido, la experiencia analizada resulta sumamente limitada, tanto en términos cuantitativos (escasa asistencia en las comisiones y talleres en relación al total poblacional, reducido número de encuentros) como en términos cualitativos. En relación a los aspectos cualitativos, la nula implementación de mecanismos tendientes a reducir las asimetrías sociales, los diferenciales de poder, los liderazgos y los intereses corporativos; así como la arbitraria división en comisiones sin debates plenarios, constituyen parte del restringido escenario participativo de la experiencia de planificación en Colón. Al respecto, resultan ilustrativos los aportes de Barrera quien refiere a los

... peligros de la fetichización instrumental de un proceso de participación que es -o debería ser- mucho más rico y complejo que una simple ingeniería administrativa"

(Barrera, 2009:114).

Asimismo, la limitada participación social expuesta puede ser interpretada como un exponente que excede las particularidades del caso, para formar parte de una problemática común a las experiencias de gestión participativa en nuestro país. En este marco interpretativo es posible, coincidiendo con las reflexiones propuestas por Robirosa (2002) considerar el caso de Colón como un ejemplo de las carencias respecto a las experiencias de gestión efectivamente participativas y a la falta de metodologías participativas probadas para la planificación y gestión urbana. Los "espacios de articulación” (Robirosa 2002: 8) han estado presentes parcialmente en la experiencia de Colón, aunque no exentos de las limitaciones respecto a la exclusión de actores sociales significativos y el manejo de las relaciones de poder.

\section{2.d) Los resultados y sus formas de producción.}

Al momento de analizar los resultados del proceso realizado en este municipio es pertinente recordar que los objetivos y resultados esperados se encontraban acotados por la redacción de un anteproyecto de ordenamiento urbano y territorial en el marco contractual y de recursos ya descripto. Considerando esto, el análisis crítico de sus resultados se limita en función de estas características. 
Realizadas estas aclaraciones, puede observarse que los principales productos de esta experiencia se materializan en un documento de informe final que incluye una propuesta de código de ordenamiento territorial con zonificación, indicadores urbanísticos y articulado con definiciones operativas precisas. Este producto remite inexorablemente a las características de planificación normativa racional presentes, parcialmente, en el proyecto. Asimismo, el carácter técnico normativo del instrumento, su concepción casi unipersonal por un especialista partícipe del equipo de investigación, su énfasis en el incremento de la densificación urbana y el control de la expansión de la ciudad parecen reforzar los aspectos físicos, racionales y normativos de la propuesta. Asimismo, queda abierta la interrogante de la injerencia que el trabajo de diagnóstico -territorial y social "participativo"- tiene en el resultado final del código.

Cabe destacar que el proyecto fue realizado con el objeto de adaptar el cuerpo normativo vigente a las etapas del proceso de ordenamiento territorial establecidas por la normativa provincial ya mencionada. En dicho contexto se procuró el desarrollo de instrumentos regulatorios para la planificación - gestión urbana y el diseño de políticas territoriales tendientes a promover el acceso a derechos urbanos, a la disminución de incompatibilidades en el uso del territorio, y la aminoración de desigualdades socioespaciales. Sin embargo, el poder regulador del instrumento de zonificación de usos del suelo y de los indicadores urbanísticos asociados, en el marco de la ley 8.912, también puede concebirse como una fuente de asignación de valor diferencial a los fragmentos del territorio, y como un mecanismo de transferencia de dicho valor, que no necesariamente garantiza los propósitos mencionados con respecto a los derechos urbanos y la disminución de asimetrías.

Por otro lado, los ejes estratégicos presentes en el documento final y las etapas propuestas para el desarrollo de grandes proyectos forman parte de la perspectiva estratégica de la planificación también presente en el caso. Sin embargo, estos lineamientos estratégicos parecen presentar fuertes limitaciones para su operativización y concreción en el mediano plazo. Estas dificultades se relacionan con algunas características intrínsecas de la jurisdicción municipal, en relación a los recursos -generalmente escasos- disponibles, a los alcances políticos limitados (proyectos de escala regional, nacional e internacional sumamente ambiciosos) y a la inexistencia de instituciones y mecanismos de gestión capaces de realizarlos. En este sentido, los lineamientos estratégicos resultantes conforman un cuerpo de buenas intenciones y deseos, en principio ajenos a las capacidades reales de un municipio.

Por último, entre los resultados de la experiencia también se encuentran presentes la propuesta de instrumentos de gestión y seguimiento del proceso de planificación del territorio municipal, algo que resulta innovador en el marco del diseño institucional del municipio de Colón y que ilustra una perspectiva de planificación que incluye, de 
manera integrada, la evaluación y gestión de los procesos. En este marco, la iniciativa de mecanismos institucionalizados de seguimiento, evaluación e implementación de los instrumentos de planificación resulta sumamente interesante. Sin embargo, es necesario mencionar ciertas tensiones potenciales entre la propuesta de este tipo de mecanismos y la ética profesional, algo que de ninguna manera es exclusivo de esta experiencia y que, por el contrario, atraviesa a la mayor parte de los proyectos de este tipo. Se hace referencia aquí a las dificultades y tensiones derivadas de la necesidad de contratar para estas instituciones recursos humanos con determinada expertise ausente en el medio local y a menudo presente en el equipo consultor.

2.e) La (des) articulación con las instancias de validación, implementación y gestión.

La planificación del territorio es más un hecho social y político que una instancia técnica; por ende, necesita de un proceso de constante renovación y actualización en un marco democrático y participativo (Clichevsky y Rofman; 1989). La propuesta realizada para Colón coincide con la apreciación de estos autores y prevé los mecanismos necesarios para cumplimentar este objetivo. No obstante, es necesario identificar ciertas limitaciones referidas a la planificación entendida como un proceso flexible, con evaluación y reformulación constantes. En este sentido, y dadas las características de esta experiencia -compartidas por muchos otros proyectos similares- debe considerarse que la lógica de la consultoría externa, la limitación de recursos, el periodo temporal acotado por contrato, el retiro de campo, la escasa transferencia de capacidades al equipo local, y la propiedad de insumos de investigación por parte del equipo consultor, limitan seriamente la revisión y actualización constante que sería deseable. Así, existe una clara frontera entre los alcances del proyecto mientras éste se desarrolla y su posterior seguimiento, revisión y validación. Sobre este último aspecto, tal como se establece en las leyes 8912/77 y 10128/83, los pasos de validación necesarios para la sanción final de la ordenanza suponen un proceso de aprobación a nivel municipal, luego a nivel de las instituciones provinciales competentes (14), y por último el Poder Ejecutivo que interviene previo dictamen del nivel provincial. El resultado de esta experiencia aún no se materializa en la sanción legal de la ordenanza municipal que permita pasar a Colón de la primera etapa de "Delimitación preliminar de áreas" a las etapas de "Zonificación" y "Planes de ordenamiento urbano", tal como la ley vigente establece. En este contexto, la constante revisión, evaluación y actualización confronta con tiempos administrativos más rígidos, lo que muchas veces redunda en instrumentaciones "de hecho" de la ordenanza y la generación de anacronismos temporales entre la ordenanza legalmente aprobada y la naturaleza fuertemente dinámica de los procesos territoriales. 


\section{Reflexiones finales}

Han sido expuestos en este trabajo elementos que permiten afirmar que la experiencia de planificación territorial analizada contiene fundamentos parcialmente contradictorios, fruto de una hibridación teórico - ideológica que impregna postulados, métodos y prácticas, no siempre en el plano explicito y/o consciente por parte de los sujetos participes del proyecto. Se han analizado elementos propios de una perspectiva neoclásica en la cual el énfasis físico, el economicisimo liberal, la ambición regulatoria y prescriptiva de las propuestas, así como la lógica racional subyacente, forman parte de una perspectiva planificadora reminiscente a la planificación normativa racional. Asimismo, algunas de las características del proyecto permiten argumentar que, aún dentro de una corriente neoclásica, son incorporados elementos propios de la planificación estratégica situacional. Los procedimientos participativos y los mecanismos de gestión previstos son ilustrativos de estas características.

Por otro lado, aparecen en la experiencia ciertos elementos vinculados a una corriente crítica en planificación, especialmente en relación a la incorporación multiactoral, a la generación de conocimiento científicamente fundado en las especificidades constitutivas del territorio investigado, al abordaje conceptual de territorio y lugar de base marxista, y al intento de una visión integral compleja, tendiente a la interdisciplinariedad y a la superación de los recortes fragmentarios de la realidad. Sin embargo, como ha sido expuesto, muchos de estos elementos constituyen sólo intentos parcialmente logrados que generan una evidente tensión en relación a la coherencia interna del proyecto. Continuando con estas apreciaciones, se entiende que

Al ser la planificación una modalidad de socialización, $y$ no un procedimiento abstracto de racionalidad humana, la discusión sobre la misma estará contextuada por la pugna fundamental entre diversas fuerzas sociales $y$ sus proyectos respectivos, así como por la relación existente entre Estado y sociedad.

(Coraggio; 1994:193).

Este principio, fundado en una perspectiva crítica de la planificación, parece haber sido omitido -o al menos mal resuelto- en el proyecto analizado; no tanto en términos discursivos sino preferentemente en relación a los mecanismos implementados y a los resultados obtenidos.

En relación a los resultados, se ha expuesto que los mismos se encuentran en el caso estudiado representados principalmente por un documento que incluye lineamientos estratégicos, indicadores urbanísticos materializados en un código de ordenamiento, 
un proceso participativo limitado y unos mecanismos de gestión interesantes pero de difícil concreción. Asimismo, las características inherentes a la lógica de un estudio de encargo realizado por un equipo consultor externo, sin transferencia ni instalación de capacidades en el ámbito local, lleva a un proceso con discontinuidades que se manifiesta en la excesiva dilatación de la sanción legal de la ordenanza. Dicha sanción permitiría instrumentar y hacer operativos los resultados de la investigación, incrementando la utilidad del estudio y el "éxito" del mismo. En ese sentido, parte de la literatura reciente que aborda otras experiencias de planificación en Argentina hace referencia a procesos con resultados "exitosos" de planificación, argumentando acerca de los beneficios de los procesos participativos, de la priorización democrática de proyectos, del incremento de la conciencia colectiva y la capacitación de los actores, a procesos de integración regional, al reposicionamiento local en un contexto regional y global competitivo, entre otros elementos. Sin negar que algunos de dichos elementos pueden ser deseables, y considerando que los procesos territoriales son sumamente complejos como para reducirlos a un exitismo ingenuo o un pesimismo fatalista, se pretende argumentar en estas reflexiones finales acerca de la escasa capacidad de transformación real que tiene una experiencia de planificación como la de Colón en las estructuras territoriales (y por ende socioeconómicas) locales. En este marco, se plantea que la experiencia no puede ser entendida como exitosa, pero que esta condición es compartida por la mayor parte de las experiencias de planificación que así se consideran. Desde el posicionamiento teórico ideológico que orienta este trabajo de revisión critica, se considera que la planificación territorial orientada por principios economicistas liberales, aún maquillada con principios democráticos y participativos, no puede ser considerada exitosa ni deseable, en la medida que contribuye, por acción u omisión, a reproducir - y en algunos casos a incrementar - las contradicciones, asimetrías y conflictos sociales.

Tal como plantea De Mattos (1993) América latina en general y Argentina en particular se han caracterizado tradicionalmente por la obstinada marginalidad de sus políticas territoriales. Sin embargo, este escenario pareciera revertirse en años recientes, aún de manera limitada, en favor de un mayor interés por la generación de políticas públicas que tienen una perspectiva planificadora con el territorio como eje central. En dicho contexto, los desafíos académicos y políticos en torno a la planificación territorial son sumamente complejos a la vez que profundamente movilizadores. Así, la revisión crítica de experiencias de planificación, la reformulación y desnaturalización de sus postulados, la deconstrucción de los principios hegemónicos en favor de propuestas alternativas, los esfuerzos en torno a la construcción de una acción transdisciplinar (Max Neef; 2004) parecen caminos productivos que seguir. En esta trama de desafíos teóricos, metodológicos y técnicos, y a partir de la premisa de que dichos desafios deben ser planteados y resueltos en el marco de proyectos ideológico - políticos en pugna, este trabajo pretende inscribirse como un humilde aporte. 
Figura 1

\section{Etapas}

Eje Temporal

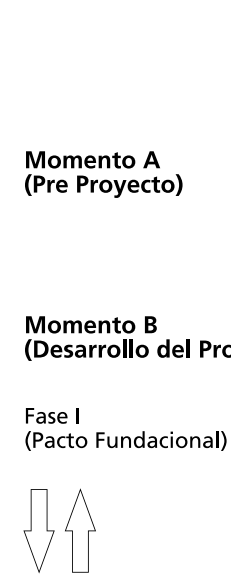

Fase II

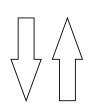

Fase III

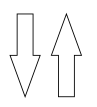

Fase IV

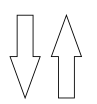

Fase V

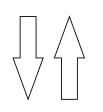

Momento C

(Post Proyecto)
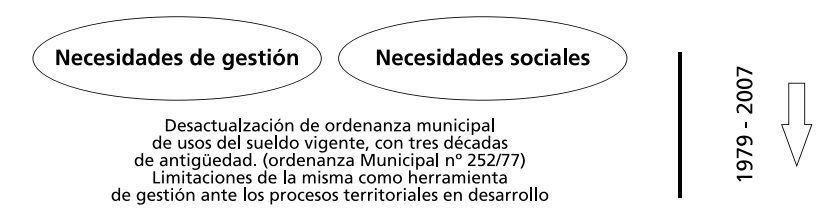

de gestión ante los procesos territoriales en desarrollo

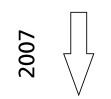

Llamado del municipio a compulsa pública

각

\section{)}

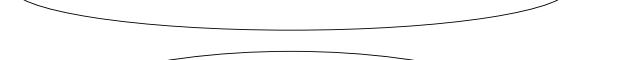

Presentación del Proyecto

Diagnóstico y constitución de comisiones

Comisiones

Técnica Política Ciudadana Empresaria

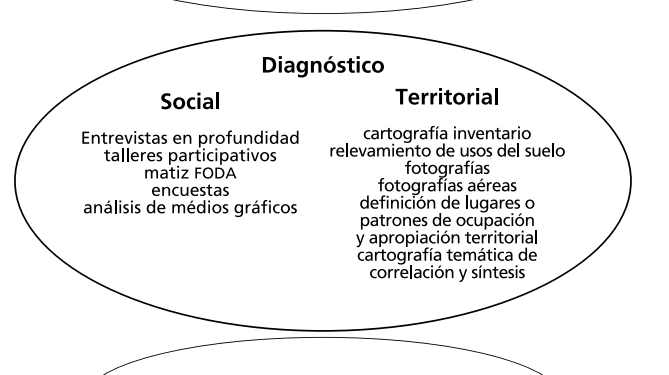

Lineamientos estratégicos

Construcción de propuesta de código
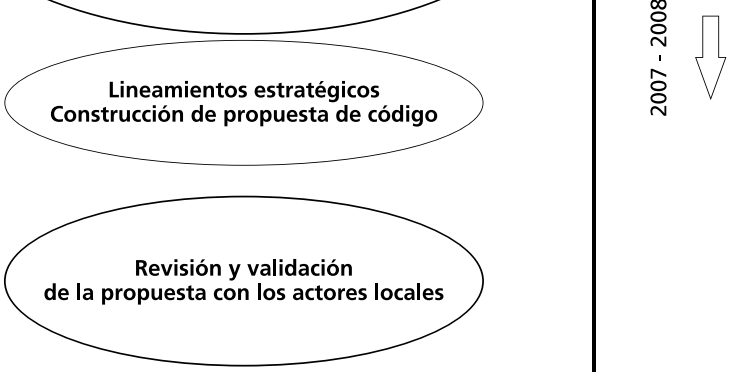

Redacción de Informe

Lineamientos estratégicos

Normativa: Código de ordenamiento territorial

Prestación Pública

Instancias de validación normativa - institucional
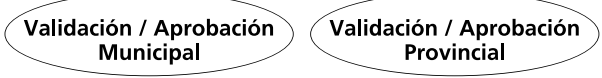

শ্㐅ে

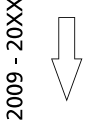

Fuente: Elaboración propia 
${ }^{1}$ Desarrollada en el marco del contrato suscrito entre la Intendencia Municipal de Colón, Buenos Aires y la Cooperativa de Profesionales Terratorium en el año 2007. Expediente Nro. 4024-170/07 caratulado "Concurso de Precios N $N^{\circ}$ 17/07- Anteproyecto de Ordenamiento Territorial y Uso del Suelo”. Los resultados del análisis crítico que en este trabajo se exponen constituyen una visión parcial, necesariamente subjetiva y personal, de exclusiva responsabilidad del autor. La revisión y valoración que se hace del proceso constituye un intento por aportar elementos al debate respecto a las potencialidades y limitaciones de la planificación territorial en Argentina, así como sobre los supuestos teórico - metodológicos subyacentes en experiencias empíricas concretas. En este sentido, estas líneas deben entenderse con fines constructivos, no debiendo interpretarse como una descalificación al proyecto ni a sus integrantes. Todos ellos quedan exentos de las apreciaciones, omisiones y errores que este trabajo pueda contener. 


\section{Referencias}

Aguilar, Luis (1992) El estudio de las políticas públicas. Estudio introductorio y edición. México: Ed. Miguel Ángel Porrua.

Aguilar, Luis (2009) "Marco para el análisis de las políticas públicas”. En Mariñez, Freddy y Garza, Vidal (2009) Política pública y democracia en América Latina del análisis a la implementación. México, D.F.: Ed. Miguel Ángel Porrua, 2009, 11-31.

Barrera, A. (2009) "Innovación política y participación ciudadana. Tendencias democráticas en los gobiernos locales". En Poggiese, H. y Tania Cohen Egler (Comps.) Otro desarrollo urbano. Ciudad incluyente, justicia social y gestión democrática. Buenos Aires: Clacso. 105-116.

Busto, C. R. (2010) “El poder entre nosotros: El Galpón de Moreno. Breve análisis del proceso de emergencia de una organización de base territorial". Revista Theomai, 21, 215-223.

Cabeza, A.M. (2012) "Políticas latinoamericanas de ordenamiento territorial. Realidad y desafios". Conferencia publicada en: Ángel Massiris Cabeza (y otros) Procesos de ordenamiento en América Latina y Colombia. Bogotá: Universidad Nacional de Colombia, 13-30.

Caminotti, Mariana. (2003) "Regionalización y gestión del desarrollo local en la provincia de Buenos Aires. La experiencia del Plan Estratégico Tuyú Mar y Campo". En Cravacuore, Daniel (coord.) Alianzas para el desarrollo local en Argentina. Experiencias, aprendizajes y desafios. Ed. Dunken, Buenos Aires. 33-58.

Catenazzi, A. y Reese, E. (2001). La construcción de estrategias de desarrollo local en las ciudades argentinas. Análisis sobre los nuevos escenarios y sus implicancias en la planificación participativa. Alcances y limitaciones en experiencias argentinas. Curso de Posgrado Desarrollo local en áreas metropolitanas, Módulo 3/ Prof. Eduardo Reese, Recuperado de: http:// www.urbared.ungs.edu.ar/textos/Mod3EReese.doc.

Chiarella Quinhoes, R. (2005) "Planificación estratégica y desarrollo territorial". Revista Espacio y Desarrollo, No 17, 208 - 225.

Cirio, G. (2012). "Principios metodológicos generales de la Inteligencia Territorial en América latina. Estado del arte de los medios de investigación de la Inteligencia Territorial en América Latina”. En Horacio Bozzano et al, Inteligencia Territorial. Teoría, métodos e iniciativas en Europa y América latina. La Plata. Edulp. 190-219. 
Clichevsky N, Rofman, Alejandro. (1989). "Planificación urbana y regional en la Argentina. Una revisión crítica”. Ciudad y territorio $N^{\circ} 79-1: 61-71$

Coraggio, José Luis (1994) Territorios en transición. Crítica a la planificación regional en América Latina. Toluca: Universidad Autónoma del Estado de México, $3^{\circ}$ ed.

De Mattos, Carlos (1993) "La obstinada marginalidad de las políticas territoriales: el caso latinoamericano". Estudios Regionales N 35. 77-114.

Elizalde Hevia, A. (2003) Planificación estratégica territorial y políticas públicas para el desarrollo local. Santiago de Chile: ILPES, serie Gestión pública.

Erbiti, C.; Guerrero, E.; Dillon, A. (2005) "El proceso de participación en el plan de ordenamiento territorial del municipio de Tandil (Argentina)". Scripta Nova. Revista electrónica de geografía y ciencias sociales. Barcelona: Universidad de Barcelona, 1 de agosto de 2005, vol. IX, 194 (97). Recuperado de: http://www.ub.es/geocrit/sn/sn-194-97.htm.

Farinós Dasí, J. (2000) “Análisis geográfico regional y planificación territorial”. Cuadernos de geografía, $\mathrm{N}^{\circ}$ 67-68:57-76.

Garay A. (2004) "Revisión crítica de los planes estratégicos desarrollados en Argentina”. En Alicia Ziccardi (coord.) Participación ciudadana y políticas sociales del ámbito local. México: Instituto de Investigaciones Sociales, UNAM.

López, M. (1999) "La traslación de estrategias empresariales al territorio: problemas de la Planificación Estratégica en el urbanismo". Política y Sociedad (Universidad de Vigo) 31:93-116.

Max-Neef, M. (2004) “Fundamentos de la transdiciplinaridad”. Valdivia, Universidad Austral de Chile. Recuperado de:http://www.max-neef.cl/download/Max_Neef_Fundamentos_transdisciplinaridad.pdf

Merklen, Denis (2004) "Sobre la base territorial de la movilización popular y sobre sus huellas en la acción” Revista Lavboratorio/online, No. 16. Buenos Aires. http://publicaciones.sociales.uba.ar/index.php/lavboratorio/index. ISSN en línea 1852-4435.

Mihura, E..; Pagnone D.; Stamatti, G.; De Carli, R.; García, F. (2013) Estrategias para el desarrollo local sustentable en la ciudad de Crespo (ER) Argentina. En VI Jornadas de la Asociación Argentino Uruguaya de Economía Ecologice. Salta, 26 al 29 de noviembre. Recuperado de http://www.asauee2013.com.ar/asaue/wp-content/ uploads/2013/11/7_2_Mihura.pdf 
Municipalidad de Colón (2008) Anteproyecto de ordenamiento territorial y usos del suelo. Municipio de Colón, Buenos Aires. Convenio Municipalidad-Cooperativa de Profesionales Terratorium. La Plata (inédito).

PECRE (2000) Plan Estratégico de Desarrollo de Crespo. Municipio de Crespo. Provincia de Entre Rios. Ordenanza CD No 24/02. 13/06/2002. 2000/ 2005. (Inédito).

Pirez, Pedro (1995) “Actores sociales y gestión de la ciudad”. Ciudades, Red Nacional de Investigación Urbana, Año 7, No. 28, octubre-diciembre, México. Disponible en: http://www.rniu.buap.mx/edit/revistas/revistas1.php.

Reese, E. (1999) Planes estratégicos: limitaciones y oportunidades. Curso internacional Ciudad futura nuevas modalidades en planificación y gestión de ciudades. Rosario, mayo de 1999. Documento de cátedra. Disponible en http://www.ceppia.com.co/Herramientas/PLANEACION-ESTRATEGICA/Reese-Eduardo-Planes-Estrategicoslimitaciones-y-oportunidades.pdf.

Reese, E. (2006) "La situación actual de la gestión urbana y la agenda de las ciudades en la Argentina". Medio Ambiente y Urbanización,Volumen 65. № 1. Instituto Internacional de Medio Ambiente y Desarrollo, IIED América Latina.Vicente López, Argentina. 3-21.

Robirosa, M. (2002) "La participación en la gestión. Justificación, malos entendidos, dificultades y estrategias". Mundo Urbano No 18 Recuperado de: http://www.mundourbano.unq.edu.ar/index.php/ano-2002/54-numero-18/79-4-la-participacion-en-lagestion-justificacion-malos-entendidos-dificultades-y-estrategias.

Santos, M. (1979) "Planejando o subdesenvolvimento e a pobreza". En Santos, M., Economía espacial: críticas e alternativas. Editorial da USP, 2003. 13-40.

Santos, M. (1996) La naturaleza del espacio. Técnica y tiempo. Razón y Emoción. Barcelona,Ariel.

Szajnberg, D; Cordara, C. y Bories, C. (2010) "Balance de las estrategias de intervención urbanística en los albores del siglo XXI". Scripta Nova. Revista Electrónica de Geografía y Ciencias Sociales. [En línea]. Barcelona: Universidad de Barcelona, 1 de agosto de 2010, vol. XIV, no 331 (24). Recuperado de: http://www.ub.es/geocrit/sn/sn-331/ sn- 331-24.htm.

Vázquez-Barquero, A. (1995) "Los planes estratégicos para el desarrollo urbano". Revista de coyuntura económica. N. 3:25-37. 
Vázquez-Barquero, A. (2009) "Desarrollo local. Una estrategia para tiempos de crisis”. Apuntes del CENES, Vol. 28, N. 47, 117-132. ISSN-e 0120-3053.

Villarreal Cantú, E. (2010) “Políticas Públicas”. En: (Pre)Textos para el análisis político. Disciplinas, reglas y procesos. México: Flacso, 257-275.

Vommaro, G. (2006) Los sectores populares y la participación en espacios de sociabilidad política locales: el Barrio Ejército Argentino (Santiago del Estero). Ponencia presentada en IV Jornadas de Investigación en Antropología Social, Buenos Aires. 